\title{
Can exercise prevent the onset of chemotherapy-induced peripheral neuropathy?
}

\author{
(c) The Author(s), under exclusive licence to Springer Nature Limited 2021
}

To date, there are no effective interventions to prevent the onset and severity of chemotherapy-induced peripheral neuropathy (CIPN). Exercising during chemotherapy treatment has displayed a range of clinical benefits, yet only limited published studies have investigated whether exercise is protective against preventing CIPN. This Editorial discusses a randomised control study of the efficacy of strength or balance exercise to prevent CIPN.

British Journal of Cancer (2021) 125:1319-1320; https://doi.org/ 10.1038/s41416-021-01489-5

Chemotherapy-induced peripheral neuropathy is a common and dose-limiting side-effect from numerous neurotoxic chemotherapies [1]. Sensory or motor CIPN symptoms can effect around half of cancer patients, with symptoms often leading to functional impairments that can impact quality of life [2]. As suggested by leading oncology institutions, there are no established interventions to prevent the onset or severity of CIPN, with dose adjustments one of the few methods at minimising symptom-burden $[3,4]$.

Exercise as an intervention has been more commonly used in oncology clinics. Established benefits include improved physical function, cardiometabolic profile, psychological wellbeing and symptom profile, as well as associations with reduced recurrence and improved survival [5]. It is unsurprising that many institutions are endorsing the promotion of exercise to patients [6].

Few studies have explored the effect of exercise for CIPN. Regular resistance, aerobic and balance exercise can be beneficial in improving the symptoms that are associated with CIPN including impaired balance, strength and functional capacity [7]. However, the preventative effects of exercise on CIPN symptomology including numbness, tingling and pain are less known. As CIPN symptoms can result in reduced quality of life due to the resulting changes in lifestyle and functional capacity [1], preventing the onset of CIPN symptoms and the associated functional side-effects are critical at improving many aspects of survivors lives.

In this issue of the British Journal of Cancer, Müller et al. present findings of the 'PIC study', a well-designed three-arm randomised control trial evaluating the preventative potential of balance or resistance exercise versus usual care on objective CIPN symptoms during neurotoxic chemotherapy. While exercise has recently been investigated in the context of treatment for already developed CIPN, few studies exist investigating exercise as a preventative therapy. Additionally and in particular, most studies lack objective and neurological assessment of CIPN, which were conducted in this study.

In this study, 170 participants (mostly female, breast cancer patients receiving taxane chemotherapy) received exercise training for $105 \mathrm{~min} /$ week throughout the duration of their chemotherapy $(17.2 \pm 5.3$ weeks). Participants were randomised to receive either: (1) Resistance Training (RT): supervised machinebased $2 \times 45 \mathrm{~min}$ at $70-80 \%$ of their maximum lifting capacity and $1 \times 15$ min home-prescribed core-strengthening session; (2) Sensorimotor (balance) Exercise Training (SMT): $3 \times 35 \mathrm{~min}$ either at home or supervised in a group setting; or (3) Usual Care (UC), which did not receive any physical activity support but was offered either intervention after completing treatment. Both intervention arms received weekly phone calls to monitor compliance and adverse events.

This study is one of the few exercise interventions during chemotherapy to include a detailed neurophysiological assessment, with many studies relying solely on clinician-graded and patientreported symptoms. While these are important tools in both clinical and research settings, high quality exercise studies including objective measures of CIPN have been lacking, particularly investigating the preventative effect. This study used the Total Neuropathy Scorereduced (TNSr), which is a composite scoring tool assessing patientreported and objective clinical CIPN symptoms including sensory, motor, deep tendon reflexes and strength. Peroneal and sural nerve conduction studies were assessed, including action potentials and nerve conduction velocities. Postural control was objectively measured using an AccuSway force plate with eyes open for maximum duration (single leg stance) and closed for 30 seconds (bipedal stance). Muscle strength was assessed using a quadriceps maximum voluntary contraction with an isokinetic dynamometer. Patientreported outcomes were used to assess CIPN (EORTC-QLQ-CIPN20), quality of life (EORTC-QLQ-C30) and fear of falling (FES-I). Assessments were conducted before and shortly after chemotherapy, as well as 3 and 6 months later.

Critically, this study did not find that either the resistance or balance exercise interventions prevented objective or subjective CIPN symptoms using intention-to-treat analyses. When excluding the high number of participants that did not adhere to the prescribed interventions, the adherent exercisers displayed reduced subjective sensory CIPN feet symptoms during chemotherapy compared to the UC group $(P=0.039, \mathrm{ES}=1.27)$. Improved selfreported sensory CIPN symptoms have been similarly reported in previous research of exercise during chemotherapy [8, 9]. Additionally, balance was preserved after completing treatment in both exercise groups compared to a reduction in the control group (SMT: $P=0.045, \mathrm{ES}=0.27 ; \mathrm{RT}: P=0.023, \mathrm{ES}=0.28)$. Although these 
findings did not persist until the follow-up timepoints, they could have important clinical ramifications on fear of falling and falls-risk that can be exacerbated due to CIPN symptoms during the critical treatment period [10].

Adherent exercisers in the study experienced benefits consistently reported in the exercise-oncology literature including improved muscle strength $(P<0.001, \mathrm{ES}=0.57)$, quality of life $(P=0.005, \mathrm{ES}=$ $0.64)$, physical function $(P=.014, \mathrm{ES}=0.63)$ and fatigue $(P=0.016$, $\mathrm{ES}=0.45)[11]$, suggesting that exercise during chemotherapy should be promoted if performed in a safe and structured manner. Adherent exercisers also had higher chemotherapy compliance $(96.6 \pm 4.8 \%$ versus $92.2 \pm 9.4 \%$ in the control group, $P=0.045)$, which could have important clinical implications due to receiving nearer to their prescribed dose. The relationship between exercise and chemotherapy dose has been investigated with a potentially beneficial effect [12], although it could be hypothesised that patients who have reduced physical function may experience more complications during treatment and are less able to exercise regularly. Yet importantly, the question regarding the preventative effect of exercise for CIPN still needs more refining.

Although the findings of this study are promising, they should still be interpreted with caution. The study initially aimed to recruit 300 participants, with the final accumulated sample size being $n=170$ given the difficulties of recruiting participants $(25 \%$ recruitment rate). Studies requiring supervised exercise interventions with additional hospital visits commencing from the beginning of chemotherapy treatment can be difficult for patients to commit to. Attendance in both interventions was around $50 \%$ (mainly due to time constraints and motivational issues), with only 35 participants across both interventions classified as adhering to $\geq 67 \%$ of the prescribed interventions, highlighting the difficulty of delivering structured exercise interventions during this period.

Exercise to prevent CIPN remains one of the few interventions that the American Society of Clinical Oncology suggests requires further clinical trials to investigate based on preliminary evidence [3]. Although studies such as by Müller et al. assist in answering this question, future studies are needed with higher adherence rates during chemotherapy to assess the preventative potential. Additionally, as it would be important clinically to prescribe a combination of aerobic, resistance and balance exercise to patients exposed to neurotoxic chemotherapy at-risk of CIPN, future studies should incorporate multi-modal exercise, including an aerobic component, which was not included in the current study and has been shown to be important for assisting patients with CIPN [7].

David Mizrahi ${ }^{1 凶}$
${ }^{1}$ Prince of Wales Clinical School, Faculty of Medicine, University of
New South Wales, Sydney, NSW, Australia.
ॠemail: d.mizrahi@unsw.edu.au

\section{REFERENCES}

1. Park SB, Goldstein D, Krishnan AV, Lin CS-Y, Friedlander ML, Cassidy J, et al. Chemotherapy-induced peripheral neurotoxicity: a critical analysis. CA Cancer J Clin. 2013;63:419-37.
2. Gordon BS, Gbadamosi B, Jaiyesimi IA. The relationship between chemotherapyinduced neuropathy and quality of life in breast cancer survivors. J Clin Oncol. 2018;36(15_suppl):e22111.

3. Loprinzi CL, Lacchetti C, Bleeker J, Cavaletti G, Chauhan C, Hertz DL, et al. Prevention and management of chemotherapy-induced peripheral neuropathy in survivors of adult cancers: ASCO Guideline Update. J Clin Oncol. 2020;0: JCO.20.01399.

4. Jordan B, Margulies A, Cardoso F, Cavaletti G, Haugnes HS, Jahn P, et al. Systemic anticancer therapy-induced peripheral and central neurotoxicity: ESMO-EONSEANO Clinical Practice Guidelines for diagnosis, prevention, treatment and follow-up. Ann Oncol. 2020;31:1306-19.

5. Cormie $\mathrm{P}$, Zopf EM, Zhang $\mathrm{X}$, Schmitz $\mathrm{KH}$. The impact of exercise on cancer mortality, recurrence, and treatment-related adverse effects. Epidemiol Rev. 2017;39:71-92.

6. Cormie $\mathrm{P}$, Atkinson M, Bucci L, Cust A, Eakin E, Hayes S, et al. Clinical Oncology Society of Australia position statement on exercise in cancer care. Med J Aust. 2018;209:184-187, https://doi.org/10.5694/mja18.00199.

7. Kanzawa-Lee GA, Larson JL, Resnicow K, Smith EML. Exercise effects on chemotherapy-induced peripheral neuropathy: a comprehensive integrative review. Cancer Nurs. 2020;43:E172-85.

8. Bland KA, Kirkham AA, Bovard J, Shenkier T, Zucker D, McKenzie DC, et al. Effect of exercise on taxane chemotherapy-induced peripheral neuropathy in women with breast cancer: a randomized controlled trial. Clin Breast Cancer. 2019;19:411-22.

9. Kleckner IR, Kamen C, Gewandter JS, Mohile NA, Heckler CE, Culakova E, et al. Effects of exercise during chemotherapy on chemotherapy-induced peripheral neuropathy: a multicenter, randomized controlled trial. Support Care Cancer. 2018;26:1019-28.

10. Kang GE, Murphy TK, Kunik ME, Badr HJ, Workeneh BT, Yellapragada SV, et al. The detrimental association between fear of falling and motor performance in older cancer patients with chemotherapy-induced peripheral neuropathy. Gait Posture. 2021;88:161-6.

11. Stout NL, Baima J, Swisher AK, Winters-Stone KM, Welsh J. A systematic review of exercise systematic reviews in the Cancer Literature (2005-17). PM R. 2017;9: S347-84.

12. Bland KA, Zadravec K, Landry T, Weller S, Meyers L, Campbell KL. Impact of exercise on chemotherapy completion rate: A systematic review of the evidence and recommendations for future exercise oncology research. Crit Rev Oncol Hematol. 2019;136:79-85.

\section{ETHICAL APPROVAL AND CONSENT TO PARTICIPATE}

No formal ethics approval was required for this manuscript, as per the local ethics committee and national guidance. No consent to participate was required.

\section{COMPETING INTERESTS}

The author declares no competing interests. He declares that he was one of three reviewers for this publication.

\section{ADDITIONAL INFORMATION}

Correspondence and requests for materials should be addressed to D.M.

Reprints and permission information is available at http://www.nature.com/ reprints

Publisher's note Springer Nature remains neutral with regard to jurisdictional claims in published maps and institutional affiliations. 